\title{
Molecular epidemiology of multidrug resistant extended spectrum beta-lactamase producing Klebsiella pneumoniae at a Jamaican hospital, 2000 - 2004
}

Nicole A Christian ${ }^{*}{ }^{\dagger}$, Karen Roye-Green ${ }^{\dagger}$, Monica Smikle $^{\dagger}$

\begin{abstract}
Background: The accurate identification of a pathogen beyond the species level is critical in epidemiological studies and investigations of nosocomial outbreaks of infection. The clonal relatedness of 66 multidrug resistant (MDR) strains of extended spectrum beta-lactamase (ESBL) producing K. pneumoniae isolated from clinical specimens from hospitalized patients at a Jamaican hospital during a 5 year period were determined by pulsed field gel electrophoresis (PFGE).

Results: A total 10 different ESBL producing K. pneumoniae genotypes designated Clones I-X were found. The most frequently occurring strains belonged to Clones I $(21 / 66,32 \%)$, II $(15 / 66,26 \%)$, III $(13 / 66,20 \%)$ and IV $(8 / 66$, $12 \%)$ which accounted for $86 \%$ (57/66) of ESBL producing K. pneumoniae strains over the 5 year period. The remaining 9 (14\%) cases of ESBL producing K. pneumoniae were due to strains of Clones $V$-X. The 4 predominant clones persisted for several years in the hospital.

Conclusions: The clonal and temporal distribution of the MDR ESBL producing K. pneumoniae strains among clinical service areas did not suggest outbreaks of the organism during the period of study. Instead the molecular epidemiology of ESBL producing K. pneumoniae at this hospital was more representative of an endemic persistence of clones of the organism with limited dissemination from patient to patient. Further studies to investigate the factors which determine the emergence and persistence of MDR ESBL producing K. pneumoniae in Jamaican hospitals and their impact on clinical and economic outcomes at such institutions would be useful.
\end{abstract}

\section{Background}

The increasing prevalence of multidrug resistant (MDR) pathogens causing nosocomial infection constitutes a major health problem [1]. Klebsiella pneumoniae ranks among the top ten organisms causing blood stream infection, pneumonia and other invasive infections in hospitalized patients in different countries [2-4]. An increasing prevalence of multidrug resistant strains of $K$. pneumoniae which possess extended spectrum beta-lactamases (ESBL) enzymes, encoded by plasmid-borne genes which confer resistance to broad spectrum cephalosporins and other antibiotics used to treat serious

\footnotetext{
* Correspondence: nicole.christian@uwimona.edu.jm

† Contributed equally

Department of Microbiology, University of the West Indies, Mona, Kingston,

Jamaica
}

infection has been widely reported [2]. Multidrug resistance contributes to unfavourable clinical outcomes, impacts the utilization of hospital resources, increases the burden of effective infection control practice and the overall health economic cost $[1,2]$.

The prevalence of ESBL producing strains of K. pneumoniae differs between countries. In the developing world a recent study from Jamaica reported that almost one-fifth of $K$. pneumoniae isolates at a tertiary referral teaching hospital were ESBL producers [5]. The presence of ESBL-producing Gram negative bacilli in hospitals in other Caribbean islands also has been reported $[6,7]$.

This study reports the clonal relationships of MDR ESBL producing K. pneumoniae at a Jamaican hospital. 


\section{Results}

The majority of the MDR K. pneumoniae isolates were from urine specimens $(31 / 66,47 \%)$, blood $(9 / 66,13 \%)$ and sputum $(7 / 66,10 \%)$. Almost a third (19/66, 29\%) were isolated from children admitted to paediatric wards while $15 \%(10 / 66)$ were from intensive care unit (ICU) patients. The remaining 37 strains were isolated from patients admitted to medical $(\mathrm{n}=15)$, surgical ( $\mathrm{n}$ $=9)$, special care nursery $(n=5)$, orthopaedic $(n=5)$ and obstetrics/gynaecological services $(\mathrm{n}=3)$.

As shown in Table 1, in addition to ceftazidime, the majority of the isolates were resistant to trimethoprim/ sulfamethoxazole $(59 / 66,89 \%)$ and the aminoglycosides (tobramycin 50/66, 76\% and gentamicin 49/66, 74\%). All $(66 / 66,100 \%)$ isolates were susceptible to meropenem.

All 66 (100\%) isolates of MDR K. pneumoniae tested positive for ESBL production in the double- disc synergy test and the E-Test ESBL screen. The E-test ESBL screen showed that all isolates $(66 / 66 ; 100 \%)$ had MIC ceftazidime and cefepime $>32 \mu \mathrm{g} / \mathrm{ml}$ and $>16 \mu \mathrm{g} / \mathrm{ml}$, respectively. The MICs were subsequently determined by the agar gel dilution method which revealed MICs ranging from $32->1024 \mu \mathrm{g} / \mathrm{ml}$ for ceftazidime and 2 $>1024 \mu \mathrm{g} / \mathrm{ml}$ for cefepime indicating ESBL production by all $(66 / 66 ; 100 \%)$ strains.

The PFGE of XbaI digests of chromosomal DNA from the 66 ESBL producing K. pneumoniae strains revealed 10 banding patterns representing 10 genotypes which were designated Clones I-X. The most frequently occurring were Clones I $(21 / 66,32 \%)$, II $(15 / 66,23 \%)$, III (13/ $66,20 \%)$ and IV $(8 / 66,12 \%)$. Multiple genotypes in comparable frequencies were isolated from specimens from various clinical service areas. The PFGE analysis of the MDR K. pneumoniae from patients admitted to different clinical service areas and the banding patterns are

Table 1 Antibiotic susceptibilities of 66 strains of multidrug resistant (MDR) extended spectrum beta lactamase (ESBL) producing K. pneumoniae, 2000-2004

\begin{tabular}{ll}
\hline Antibiotic & Susceptibility (\%) \\
\hline Nalidixic Acid & 82 \\
\hline Norfloxacin & 88 \\
\hline Ciprofloxacin & 91 \\
\hline Levofloxacin & 85 \\
\hline Gentamicin & 26 \\
\hline Tobramycin & 24 \\
\hline Minocycline & 59 \\
\hline Nitrofurantoin & 9 \\
\hline Trimethoprim/sulfamethoxazole & 11 \\
\hline Ceftazidime & 0 \\
\hline Cefepime & 0 \\
\hline Meropenem & 100 \\
\hline
\end{tabular}

shown in Figures 1, 2, 3 and 4. There were 8 cases of MDR K. pneumoniae infection in long stay patients at the hospital. Among these, coinfections with multiple genotypes of MDR K. pneumoniae were observed in 2 admissions in ICU and Paediatrics as shown in Figure 1 (lanes 10 and 11) and Figure 3 (lanes 7 and 8), respectively. Repeat infections occurred in 2 re-admissions after 3 months and 18 months. In the first case, a different clone was involved while in the other the same clone was identified (shown in Figure 3 lanes 2 and 3).

The temporal distribution of the ESBL producing $K$. pneumoniae clones among various hospital services over the 5 year period is summarized in Table 2 . There were 7 ESBL producing K. pneumoniae isolates during 2000, 12 during 2001, 30 during 2002 and 12 and 5 isolates during 2003 and 2004, respectively. The MDR ESBL $K$. pneumoniae strains belonging to Clones I, II, III and IX were isolated from patients in 4 different clinical service areas during 2000. Clones I and II were first identified in infants on the paediatric wards during July and August and Clone I in 2 patients on the medical wards during September of that year. Clones I-IV were present in the hospital during 2001 with multiple genotypes occurring in 3 of the 6 clinical service areas. The increased prevalence of ESBL producing $K$. pneumoniae observed in the hospital during 2002 involved strains belonging to Clones I-IV. However all 7 clinical service areas were affected but no new genotypes were identified in that year. In contrast the subsequent decline in the frequency of isolates during 2003 was accompanied by the emergence of new genotypes including Clones VVIII which were identified in clinical specimens from 3 ICU patients and the reemergence of clone I in the hospital after an absence of 10 months. During 2004 3 of 5 isolates from patients admitted to Surgery and Paediatrics belonged to Clone VI.

As shown in Table 3, based on the antibiotic susceptibility testing 13 antibiotypes (R1-R13) were identified. There were 22 (33\%) quinolone-resistant isolates which were assigned antibiotypes R1-R7. The isolates assigned antibiotype $\mathrm{R} 1$ were resistant to all the quinolones tested. The remaining 44 isolates were quinolone sensitive and were assigned antibiotypes R8-R13. No correlations were found between the antibiotypes and genotypic clones of the MDR ESBL producing K. pneumoniae. The strains which had similar antibiotypes often belonged to different PFGE clones. However, all 6 isolates with quinolone-sensitive antibiotypes R9 and R13 belonged to PFGE Clone 1 as shown in Table 3.

\section{Discussion}

The clonal and temporal distributions of the MDR ESBL producing K. pneumoniae strains among clinical service areas in the hospital do not suggest outbreaks of the 


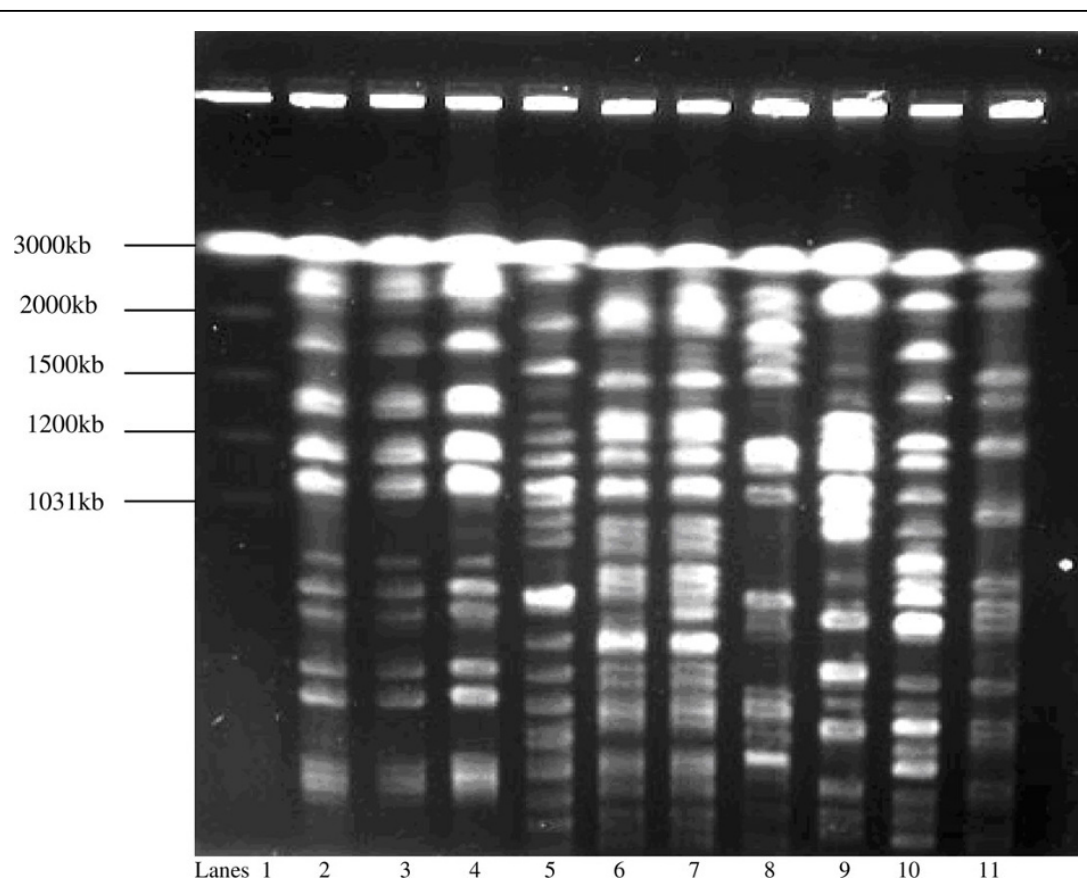

Figure 1 Pulsed field gel electrophoresis (PFGE) analysis of Xbal digests of multidrug resistant (MDR) $K$. pneumoniae strains from intensive care unit (ICU) patients (2000-2004). Lane 1: molecular size marker, Saccharomyces cerevisiae; lanes 2-4: MDR K. pneumoniae Clone I isolated during 2001; lane 5: Clone II isolated during 2002; lanes 6-7: K. pneumoniae strains belonging to Clones III, isolated 2 weeks apart from the same patient; lanes 8-9: Clones V and VI isolated in 2003; lanes 10-11: Clones VII and VIII, respectively isolated from the same patient during 2003.

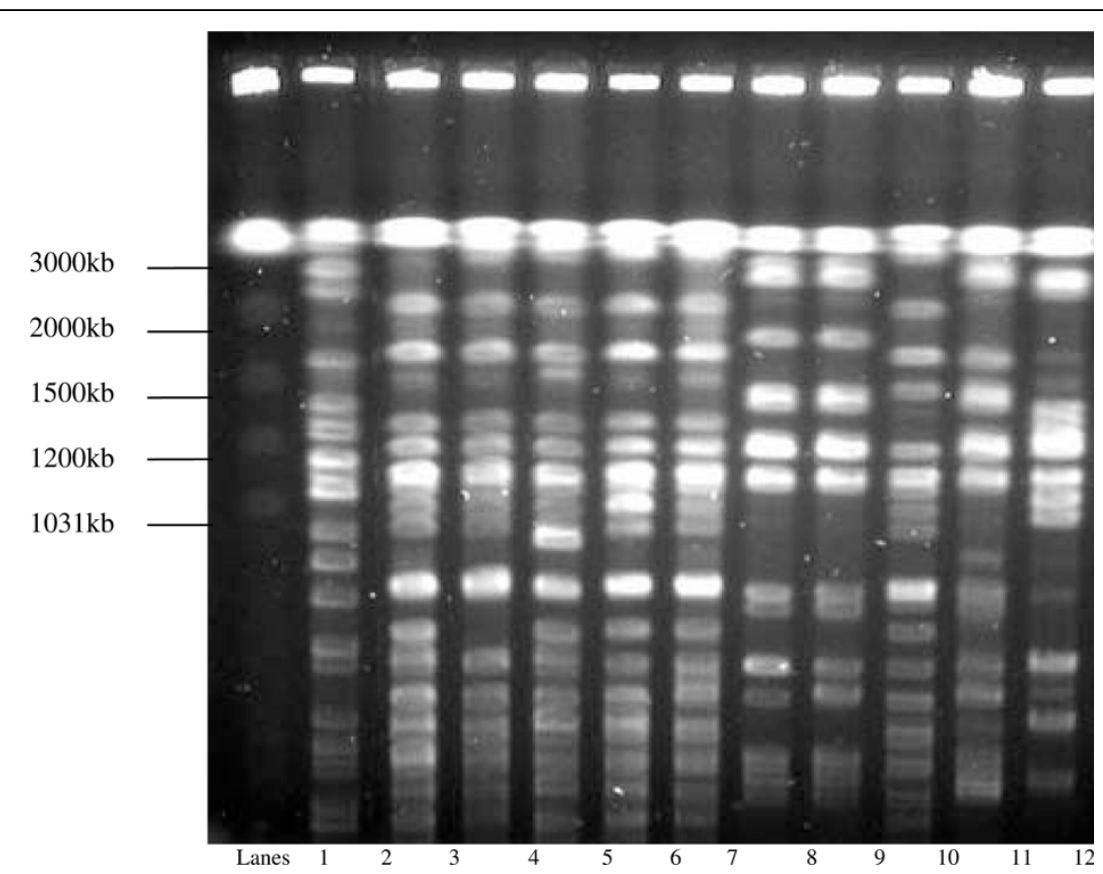

Figure 2 Pulsed field electrophoresis (PFGE) analysis of Xbal digests of multidrug resistant (MDR) K. pneumoniae strains isolated from paediatric patients (2000-2004). Lane 1: molecular size marker, Saccharomyces cerevisiae; lane 2: K. pneumoniae Clone III isolated during 2001; lanes 3-7: five strains of $K$. pneumoniae Clone II isolated from specimens collected from the same patient during the same day; lanes 8-9: Clone I isolated from unrelated patients during 2002; lane 10: Clone II isolated during 2002; lane 11: Clone I isolated during 2003 and lane 12: Clone VI isolated during 2004. 


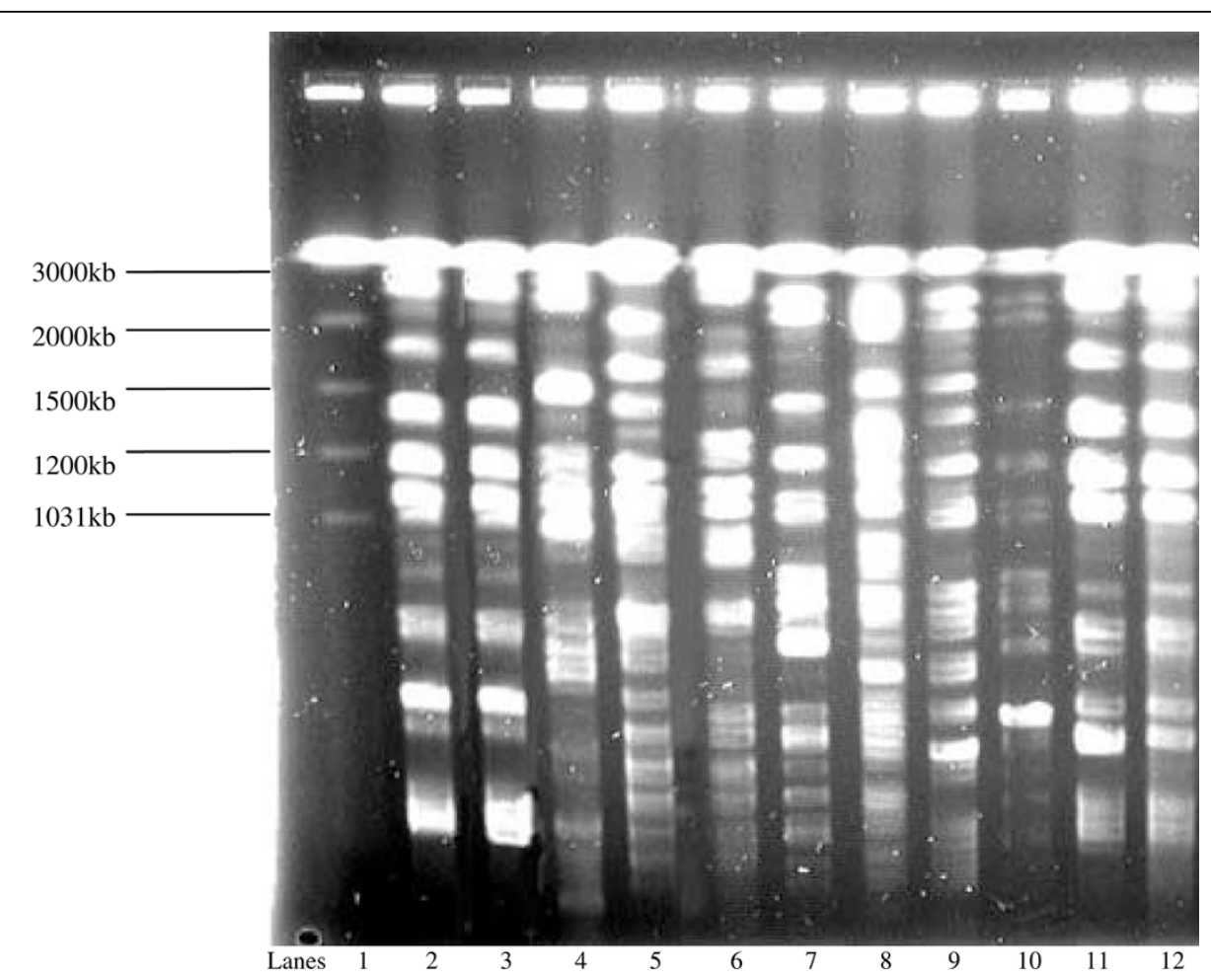

Figure 3 Pulsed field electrophoresis (PFGE) analysis of Xbal digests of 11 multidrug resistant (MDR) K. pneumoniae strains isolated from patients admitted to the paediatric wards (2000-2004). Lane 1: molecular size marker, Saccharomyces cerevisiae; lanes 2-3: two strains of MDR K. pneumoniae clone I isolated from the same patient during 2001 and 2002, respectively; lane 4: MDR K. pneumoniae clone III isolated during 2001; lanes 5-6: clone II; lanes 7-8: clones IV and III from the same patient during the same admission in 2002; lanes 9-10: clone IV; and lanes 11-12: clone I strains from different patients.

organism at that institution during the period studied. Instead the epidemiology of ESBL producing K. pneumoniae at this hospital is more representative of an endemic persistence of clones of the organism with limited dissemination from patient to patient. However, the persistence of related clones over the time period suggests patient to patient transmission or healthcare worker to patient transmission. The emergence and reemergence of Clone I in the ICU during a 6-month period during 2001 is consistent with this concept. Also the results suggest that there has been transmission of some common strains including PFGE Clones I, II and III, over time in the hospital during 2000-2004. This might have been more evident if asymptomatic patients had been screened for MDR K. pneumoniae colonization. The presence of asymptomatically colonized patients may explain the intermittent appearances of certain strains over time in various hospital services. The epidemiology of ESBL producing $K$. pneumoniae at this hospital proved complex and, as explained by Branger et al [8], may involve the spread of self-transferable plasmids as well as clonal spread [8].

Studies conducted in hospitals elsewhere have reported the spread of single clones of MDR K. pneumoniae among patients hospitalized over protracted periods of time [8,9]. In the present study ESBL producing $K$. pneumoniae strains belonging to Clone III persisted in the hospital over the 5-year period studied. During 2002 the year in which the largest number of cases, especially of paediatric cases, was seen different genotypes of the organism coexisted in patients on the same wards. This makes it less clear whether or not outbreaks caused by single different strains or involving the 4 endemic clones in the hospital had occurred. The prevalence of ESBL producers at the University Hospital of the West Indies for that year was 18\% [5]. The factors contributing to the increasing incidence of ESBL producing $K$. pneumoniae during 2002 have not been clearly defined at this hospital [5]. A number of risk factors for increased colonization with MDR K. pneumoniae including the use of third generation cephalosporins have been reviewed [10].

Other interesting observations from the study include the cases of long stay and repeat patients who remained colonized or had repeat infections with the same genotype after long periods of time and those with concomitant infections with different genotypes of ESBL producing K. pneumoniae. Branger et al [8] reported the case of a patient colonized with the same ESBL 


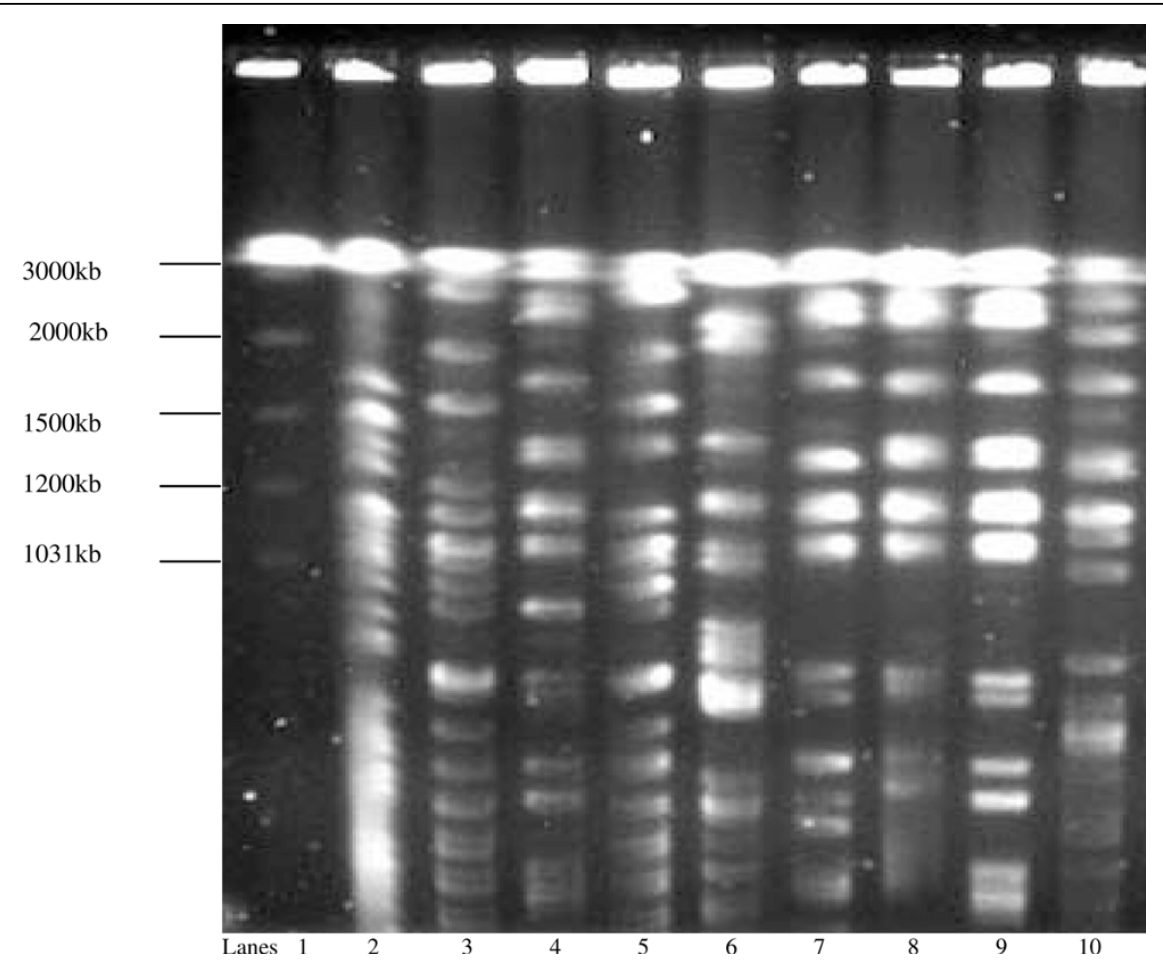

Figure 4 Pulsed field electrophoresis (PFGE) analysis of Xbal digests of 9 multidrug resistant (MDR) K. pneumoniae strains (2000-2004). Isolates were obtained from patients admitted to the orthopaedic ward (lanes 2-6) showing PFGE patterns corresponding to clone IX (lane 2), clone II (lanes 3 and 5), clone I (lane 4) and clone IV (lane 6), 2000-2002; and the medical wards (lanes 7-10) showing PFGE patterns of clone I (lanes 7-9) and clone II (lane 10), 2002-2003.

producing K. pneumoniae strain for 10 months [8]. Sequential or simultaneous isolation of unrelated strains of ESBL producing K. pneumoniae from individual patients has been reported by others [11]. Weller et al [12] reported that multiple subvariants of a strain could persist in an infective population without any one subvariant becoming dominant $[11,12]$.

The previously reported decreased susceptibility to aminoglycosides, fluoroquinolones and trimethoprim/ sulfamethoxazole in ESBL producing $K$. pneumoniae was also observed in this study [13]. The data on antibiotypes provided additional evidence in support of the clonality of the PFGE genotypes. The predominant ESBL producing $K$. pneumoniae genotypes I, II, III and
IV had the quinolone-resistant antibiotype R1. This might have contributed to the endemic persistence of these clones in the hospital [14]. The more susceptible clones were probably not endemic strains at the UHWI and may have been harboured by patients who were already colonized on admission [8]. Notably, the PFGE genotypes V, VII and VIII isolated from ICU patients also had the more resistant antibiotype R1 though found in lower numbers. A number of factors including aggressive antibiotic therapy, prolonged hospitalization and the performance of invasive procedures are well documented contributors to the increased risk of infection with nosocomial strains of MDR K. pneumoniae in patients admitted to the ICU [15].

Table 2 Temporal distribution of multidrug resistant (MDR) extended spectrum beta-lactamase (ESBL) producing $K$. pneumoniae clones by hospital service during 2000-2004.

\begin{tabular}{llll}
\hline Year & $\begin{array}{l}\text { Number of } \\
\text { Isolates }\end{array}$ & $\begin{array}{l}\text { Clone/genotypes } \\
\text { identified }\end{array}$ & Hospital Service \\
\hline 2000 & 7 & I, II, III, IX & Paediatrics, Medicine, Orthopaedics, Obstetrics \& Gynaecology \\
\hline 2001 & 12 & I, II, III, IV & $\begin{array}{l}\text { Intensive care unit, Paediatrics, Surgery, Special Care Nursery, Orthopaedics, Obstetrics \& } \\
\text { Gynaecology }\end{array}$ \\
\hline 2002 & 30 & I, II, III, IV & Intensive care unit, Paediatrics, Medicine, Surgery, Special Care Nursery, Orthopaedics \\
\hline 2003 & 12 & I, II, III, IV, V, VI, VII, VIII, X & Intensive care unit, Paediatrics, Medicine, Surgery, Special Care Nursery \\
\hline 2004 & 5 & III, IV, VI & Paediatrics, Surgery \\
\hline
\end{tabular}


Table 3 The antibiotypes and pulsed field gel electrophoresis (PFGE) clones of the 66 multidrug resistant (MDR) extended spectrum beta-lactamase producing (ESBL) $K$. pneumoniae strains, 2000-2004

\begin{tabular}{lll}
\hline Antibiotypes $(\mathbf{n})^{*}$ & Resistance Profile + & Clones of ESBL K. pneumoniae \\
\hline R1 (9) & NA, Nor, Cip, Lev, Cn, Tob, Min, F, SXT & I, II, III, VIII \\
\hline R2 (1) & NA, Nor, Cip, Lev, Cn, Tob, Min, SXT & VI \\
\hline R3 (3) & NA, Nor, Cip, Lev, Cn, Tob, SXT & III, VII \\
\hline R4 (3) & Lev, Cn, Tob, Min, F, SXT & I, II, IV \\
\hline R5 (5) & NA, Cn, Tob, F, SXT & I, II \\
\hline R6 (1) & NA, Cn, Tob, SXT & II \\
\hline R7 (1) & Lev, F & I \\
\hline R8 (2) & Min, Cn & I, II \\
\hline R9 (3) & F & I \\
\hline R10 (6) & SXT & I, II, III, IV, VI \\
\hline R11 (15) & Tob, SXT & IIIIII, IV, VI \\
\hline R12 (14) & Cn, Tob, F, SXT & III, IV, IX, X \\
\hline R13 (3) & Cn, Tob, Min, F, SXT
\end{tabular}

${ }^{*} \mathrm{n}$ is the total number of MDR K. pneumoniae assigned to each antibiotype

† NA nalidixic acid, Nor norfloxacin, Cip ciprofloxacin, Lev levofloxacin, Cn gentamicin, Tob tobramycin, Min minocycline, F nitrofurantoin, SXT trimethoprim sulfamethoxazole

Clearly different antibiotic susceptibility patterns distinguish different strains of ESBL producing K. pneumoniae as shown in the current study. However, antibiotic susceptibility testing has relatively limited utility as a typing system in epidemiologic studies not only because of phenotypic variation but also because antibiotic resistance is under extraordinary selective pressure in contemporary hospitals [14]. The selective pressure from antimicrobial therapy may alter the antimicrobial susceptibility profile of an organism, such that related organisms show different resistance profiles [16]. Graffunder et al [10] found a correlation between the selective pressure of antimicrobial agents identified as risk factors for ESBL producing organisms and the presence of related resistance genes residing on the plasmids [10]. Woodford et al [16] also suggests that antibiotic pressure may have been a factor for initial colonization of patients and the development of further resistance by the organism [16].

The limitations of the study are those attending studies involving retrospective data collection, the disproportionately small number of ESBL producing $K$. pneumoniae strains from some clinical service areas, the long time period over which the isolates were collected, the lack of surveillance cultures to detect asymptomatic, colonized patients with MDR ESBL producing $K$. pneumoniae and the limited available epidemiologic data to compare with the PFGE typing results. During the extended period of study advances in medical technology, changes in patient population, formulary restrictions and changes in standards of practice or infection control measures may affect the results [10].

\section{Conclusions}

In summary the results showed clonal diversity of MDR ESBL producing $K$. pneumoniae, elements of its temporal distribution which were suggestive of endemic persistence and dissemination of this organism between patients at this hospital, the extent of which was not fully ascertained. Further studies which investigate the factors which determine the emergence and persistence of ESBL producing K. pneumoniae in Jamaican hospitals and the impact on clinical and economic outcomes at such institutions would be useful.

\section{Methods}

\section{Microbiological Investigations}

All clinical isolates $(\mathrm{n}=66)$ of MDR $K$. pneumoniae from clinical specimens collected from 57 patients admitted to the University Hospital of the West Indies (UHWI), Kingston, Jamaica, a tertiary care referral centre, between June 2000 and April 2004 were stored in tryptose soy broth at $-70^{\circ} \mathrm{C}$ until required. Standard microbiological procedures were followed for the different clinical specimens [17]. Bacterial isolates were identified and the initial antibiotic susceptibility testing was done using the Vitek automated system (Biomerieux, Durham, North Carolina, U.S.A.). The appropriate antibiotic panel for each type of specimen was used as recommended by the manufacturer. The breakpoints for antibiotic susceptibility were determined according to the guidelines of the Clinical and Laboratory Standards Institute (CLSI) [17]. The antibiotics tested included amoxicillin/clavulanic acid, ampicillin, carbenicillin, cefazolin, ceftriaxone, cefuroxime, cephalothin, ceftazidime, ciprofloxacin, gentamicin, levofloxacin, minocycline, 
nalidixic acid, nitrofurantoin, norfloxacin, ticarcillin/clavulanic acid, tobramycin, trimethoprim/sulfamethoxazole and meropenem. The MDR strains of $K$. pneumoniae were classified as organisms showing resistance to at least three classes of antibiotics including ceftazidime [18]. Resistance to ceftazidime identified by Vitek was used as the initial screening test for the presence of ESBL which was confirmed by E-test (AB Biodisk, Solna, Sweden) and double-disc synergy test which were performed according to the manufacturer's instructions and CLSI guidelines [17], respectively. A positive double disc synergy test was defined as enhancement of the zones of inhibition for ceftazidime and cefotaxime in the presence of clavulanic acid.

The MDR ESBL producing K. pneumoniae strains were assigned antibiotypes based on their resistance patterns.

\section{Pulsed Field Gel Electrophoresis}

Pulsed-field gel electrophoresis (PFGE) was used to determine the relatedness of the ESBL producing strains of $K$. pneumoniae. The PFGE was performed as described previously with modifications [19]. Electrophoresis was carried out in $0.5 \times$ TBE buffer using the Chef Mapper XA pulsed field electrophoresis system (Biorad, Hercules, California, U.S.A.). The conditions were $6 \mathrm{~V} / \mathrm{cm}$ for $21 \mathrm{~h}$ at $12^{\circ} \mathrm{C}$, with the pulse time ramped linearly from $1 \mathrm{~s}$ to $40 \mathrm{~s}$. The molecular size marker included for comparison was Saccharomyces cerevisiae (Biorad, Hercules, California, U.S.A.). Following electrophoresis the gels were stained with ethidium bromide and photographed under ultraviolet light. The banding patterns were compared based on the criteria described by Tenover et al [20]. Isolates were considered indistinguishable if their restriction patterns had the same number of corresponding bands of the same apparent size and closely related for differences of 3 bands. Isolates which differed by 4 or more bands were considered unrelated.

The study was approved by the Ethics Committee in the Faculty of Medical Sciences of the University of the West Indies, Mona.

\section{Acknowledgements}

We thank Mrs Lois Rainford, Mrs Charmaine Parkes and our colleagues in the Bacteriology Section of the Microbiology Department, University of the West Indies for their assistance.

\section{Authors' contributions}

NAC carried out the microbiological and molecular studies and drafted the manuscript. KRG and MS conceived of the study, participated in its design and coordination. All authors read and approved the final manuscript.

Received: 10 February 2009

Accepted: 28 January 2010 Published: 28 January 2010
References

1. Blot S, Depuydt P, Vandewoude K, DeBacquer D: Measuring the impact of multidrug resistance in nosocomial infection. Curr Opin Infect Dis 2007, 20:391-396, Review.

2. Marra AR, Wey SB, Castelo A, Gales AC, Cal RG, Filho JR, Edmond MB, Pereira CA: Nosocomial bloodstream infections caused by Klebsiella pneumoniae: impact of extended-spectrum beta-lactamases (ESBL) production on clinical outcome in a hospital with high ESBL prevalence. BMC Infect Dis 2006, 6:24.

3. Pfaller MA, Jones RN, Doern GV, Kugler K: Bacterial pathogens isolated from patients with bloodstream infection: frequencies of occurrence and antimicrobial susceptibility patterns from the SENTRY antimicrobial surveillance programme (United States and Canada 1997). Antimicrob Agents Chemother 1998, 42:1762-1770.

4. Gales AC, Bolmstrom A, Sampaio J, Jones RN, Sader HS: Antimicrobial susceptibility of Klebsiella pneumoniae producing extende-spectrum beta-lactamases (ESBL) isolated in hospitals in Brazil. Braz J Infect Dis 1997, 1:196-203.

5. Nicholson AM, Gayle P, Roye-Green K: Extended spectrum beta-lactamase producing organisms at the University Hospital of the West Indies. West Indian Med J 2004, 53:104-108.

6. Orett FA: Resistance patterns among selective Gram-negative bacilli from an intensive care unit in Trinidad West Indies. Saudi Med J 2004, 25:478-483.

7. Del Carmen Rodriguez M, Vera DE, Ramirez-Ronda CH, Saavedra S: Phenotypic confirmation of extended-spectrum B-lactamases (ESBL) in clinical isolates of Escherichia coli and Klebsiella pneumoniae at the San Juan Veterans Affairs Medical Center. P R Health Sci J 2004, 23:207-215.

8. Branger C, Lesimple AL, Bruneau B, Berry P, Lambert-Zechovsky N: Longterm investigation of the clonal dissemination of Klebsiella pneumoniae isolates producing extended-spectrum $\beta$-lactamases in a university hospital. J Med Microbiol 1998, 47:201-209.

9. Bingen EH, Desjardins P, Arlet $G$, Bourgeois F, Mariani-Kurkdjian P, LambertZechovsky NY, Denamur E, Philippon A, Elion J: Molecular epidemiology of plasmid spread among extended-broad spectrum $\beta$-lactamaseproducing Klebsiella pneumoniae isolates in a pediatric hospital. J Clin Microbiol 1993, 31:179-184.

10. Graffunder EM, Preston KE, Evans AM, Venezia RA: Risk factors associated with extended- spectrum beta-lactamase-producing organisms at a tertiary care hospital. J Antimicrob Chemother 2005, 56:139-145.

11. Essack SY, Hall LM, Pillay DG, Mcfadyen ML, Livermore DM: Complexity and diversity of Klebsiella pneumoniae strains with extended-spectrum $\beta$ lactamases isolated in 1994 and 1996 at a teaching hospital in Durban, South Africa. Antimicrob Agents Chemother 2001, 45:88-95.

12. Weller TM, MacKenzie FM, Forbes KJ: Molecular epidemiology of a large outbreak of multiresistant Klebsiella pneumoniae. J Med Microbiol 1997, 46:921-926.

13. Rahal JJ, Urban C, Horn D, Freeman K, Segal-Maurer S, Maurer J, Mariano N, Marks S, Burns JM, Dominick D, Lim : Class restriction of cephalosporin use to control total cephalosporin resistance in nosocomial Klebsiella. JAMA 1998, 280:1233-1237.

14. Bedenic B, Schmidt H, Herold S, Monaco M, Plecko V, Kalenic S, Katic S, Skrlin-Subic J: Epidemic and endemic spread of Klebsiella pneumoniae producing SHV-5 beta-lactamase in Dubrava University Hospital, Zagreb, Croatia. J Chemother 2005, 17:367-375.

15. Lucet JC, Decré D, Fichelle A, Joly-Guillou ML, Pernet M, Deblangy C, Kosmann MJ, Régnier B: Control of a prolonged outbreak of extended spectrum beta-lactamase-producing Enterobacteriaceae in a university hospital. Clin Infect Dis 1999, 29:1411-1418.

16. Woodford N, Tierno PM Jr, Young K, Tysall L, Palepou MF, Ward E, Painter RE, Suber DF, Shungu D, Silver LL, Inglima K, Kornblum J, Livermore D: Outbreak of Klebsiella pneumoniae producing a new carbapenem-hydrolysing class A beta-lactamase, KPC-3, in a New York Medical Center. Antimicrob Agents Chemother 2000, 48:4793-4799.

17. Clinical and Laboratory Standards Institute: Performance standards for antimicrobial disk susceptibility tests Clinical and Laboratory Standards Institute, Wayne, Pa 2006, Approved standard M2-A9.

18. D'Agata EM: Rapidly rising prevalence of nosocomial multidrugresistance, Gram-negative bacilli: a 9-year surveillance stud. Infect Control Hosp Epidemiol 2004, 25:842-846. 
19. Birren B, Lai E: Pulsed field gel electrophoresis: a practical guide California: Academic press 1993.

20. Tenover FC, Arbeit RD, Goering RV, Mickelsen PA, Murray BE, Persing DH, Swaminathan B: Interpreting chromosomal DNA restriction patterns produced by pulsed-field gel electrophoresis: criteria for bacterial strain typing. J Clin Microbiol 1995, 33:2233-2239.

doi:10.1186/1471-2180-10-27

Cite this article as: Christian et al:: Molecular epidemiology of multidrug resistant extended spectrum beta-lactamase producing Klebsiella pneumoniae at a Jamaican hospital, 2000 - 2004. BMC Microbiology 2010 $10: 27$

Submit your next manuscript to BioMed Central and take full advantage of:

- Convenient online submission

- Thorough peer review

- No space constraints or color figure charges

- Immediate publication on acceptance

- Inclusion in PubMed, CAS, Scopus and Google Scholar

- Research which is freely available for redistribution

Submit your manuscript at www.biomedcentral.com/submit 